\title{
Atividade antiviral de Musa acuminata Colla, Musaceae
}

\section{Fernanda Otaviano Martins, ${ }^{1}$ Catharina Eccard Fingolo, ${ }^{* 2,3}$ Ricardo Machado Kuster, ${ }^{2,3}$ Maria Auxiliadora Coelho Kaplan, ${ }^{2,3}$ Maria Teresa Villela Romanos ${ }^{1}$}

\author{
${ }^{I}$ Departamento de Virologia, Instituto de Microbiologia Paulo de Góes, Centro de Ciências da Saúde, Bloco I, ss, \\ sala 064, Universidade Federal do Rio de Janeiro, Cidade Universitária, 21941-590 Rio de Janeiro-RJ, Brasil \\ ${ }^{2}$ Programa de Biotecnologia Vegetal, Pós Graduação em Biotecnologia Vegetal, Centro de Ciências da Saúde, \\ Bloco K $2^{\circ}$ andar, sala 032, Universidade Federal do Rio de Janeiro, \\ Cidade Universitária, 21941-590 Rio de Janeiro-RJ, Brasil \\ ${ }^{3}$ Núcleo de Pesquisas de Produtos Naturais, Centro de Ciências da Saúde, Bloco H, $1^{\circ}$ andar, sala 016, Universidade \\ Federal do Rio de Janeiro, Cidade Universitária, 21941-590 Rio de Janeiro-RJ, Brasil.
}

\begin{abstract}
RESUMO: O presente trabalho avalia a atividade antiviral de extratos e frações de Musa acuminata Colla, Musaceae, coletada em duas regiões do Estado do Rio de Janeiro (Petrópolis e Santo Antônio de Pádua). As inflorescências de $M$. acuminata apresentaram excelente atividade para os dois vírus avaliados: herpesvírus simples humano tipo 1 e herpesvírus simples humano tipo 2, ambos resistentes ao Aciclovir. Os resultados indicam que os extratos de M. acuminata testados podem constituir alvo potencial para uso em terapias antivirais.
\end{abstract}

Unitermos: Musa acuminata, Musaceae, atividade antiviral.

\begin{abstract}
Antiviral activity of Musa acuminata Colla, Musaceae." This study evaluates the antiviral activity of extracts and fractions of Musa acuminata Colla collected in two regions of Rio de Janeiro State (Petrópolis and Santo Antônio de Pádua). The inflorescences of M. acuminata showed excellent activity for the two virus evaluated: simple human herpesvirus type 1 and simple human herpesvirus type 2, both resistant to Acyclovir. The results indicate that the tested extracts of M. acuminata can be potential target for use in antiviral therapy.
\end{abstract}

Keywords: Musa acuminata, antiviral activity.

\section{INTRODUÇÃO}

A espécie Musa acuminata Colla, conhecida popularmente como banana ouro, pertence à família Musaceae e é considerada a menor das bananas. A banana (Musa sp.) é uma das frutas mais consumidas no mundo, sendo cultivada na maioria dos países tropicais. No Brasil, a banana destaca-se entre os principais produtos agrícolas, ocupando o segundo lugar dentre as frutas na preferência dos consumidores (Bernardi et al., 2004).

De acordo com a literatura vigente, algumas espécies de Musa caracterizam-se pela presença de substâncias com potencial farmacológico bastante interessante, tais como: antioxidante (Kanazawa \& Sakakibara, 2000; Someya et al., 2002), antiúlcera (Ghosal \& Saini, 1984; Costa \& Brito, 1997; Lewis et al., 1999), bactericida (Sharma et al., 1989), fungicida (Talero \& Vejarano, 1973; Sharma et al., 1989; Hirai et al., 1994; Quinines et al., 2000; Kamo et al., 2001; Luque-Ortega et al., 2004), inseticida (Pascual-Villalobos \& Rodriguez, 2006).

Este trabalho visa mostrar a atividade antiviral de Musa acuminata, nunca antes descrita na literatura.

\section{MATERIAL E MÉTODOS}

\section{Material vegetal}

O material vegetal foi coletado no município de Petrópolis-RJ em novembro e dezembro de 2006 e em Santo Antônio de Pádua-RJ em novembro de 2006, identificado pelo Prof. Dr. João Marcelo de Alvarenga Braga, Jardim Botânico-RJ e depositado no Herbário da Faculdade de Formação de Professores por Ricardo de Moura Loyola, Universidade Estadual do Rio de Janeiro (UERJ), São Gonçalo, Rio de Janeiro, Brasil.

As inflorescências de Musa acuminata, ainda frescas, foram reduzidas a pequenos fragmentos utilizando faca e, em seguida, submetidas ao processo de extração exaustiva por maceração estática com etanol, o solvente tendo sido trocado vinte vezes, de $48 \mathrm{em} 48 \mathrm{~h}$. O material foi concentrado sob pressão reduzida em rotaevaporador, mantendo a temperatura do banho perto de $40{ }^{\circ} \mathrm{C}$. Após secagem, o resíduo vegetal foi submetido à partição líquido-líquido em seqüência com solventes de diferentes polaridades: hexano, diclorometano, acetato de etila e 
n-butanol. As frações obtidas foram concentradas sob pressão reduzida, pesadas e então usadas nos testes de atividade antiviral.

Os extratos e as frações foram pesados separadamente e, posteriormente, solubilizados em $1 \%$ de DMSO e adicionados de água destilada para uma concentração estoque de $400 \mu \mathrm{g} / \mathrm{mL}$, filtrados em membrana Millipore $(0,22 \mathrm{~mm})$, divididos em pequenos volumes e estocados a temperatura de $-20^{\circ} \mathrm{C}$ até o momento de utilização.

\section{Células e vírus}

Para a realização desse estudo foi utilizada a cultura de células Vero (fibroblasto de rim de macaco Cercopitheccus aethiops), mantida em MEM (Meio Mínimo Essencial) suplementado com 10\% de soro fetal bovino. As amostras de vírus Herpes simplex tipos $1 \mathrm{e}$ 2 resistentes ao Aciclovir (HSV-1-ACVr e HSV-2-ACVr), pertencem à coleção do LEDAC (Laboratório Experimental de Drogas Antivirais e Citotóxicas), sob a responsabilidade da Professora Dra. Maria Teresa Villela Romanos.

\section{Testes de citotoxidade}

A citotoxidade foi determinada baseando-se na observação da alteração morfológica e na viabilidade celular.

\section{Observação da alteração morfológica celular}

Os extratos e frações foram submetidos a diluições seriadas, na razão 2 , utilizando-se meio de cultura sem soro como diluente e colocados em contato com as monocamadas de células confluentes em microplacas de 96 poços e posteriormente incubadas a 37 ${ }^{\circ} \mathrm{C}$ em ambiente com $5 \%$ de $\mathrm{CO}_{2}$ durante $48 \mathrm{~h}$ e examinada ao microscópio óptico invertido. $\mathrm{O}$ efeito citotóxico foi detectado pelo aparecimento de células morfologicamente alteradas (Rodriguez et al., 1990). A maior concentração da substância em que não houve alteração na morfologia celular foi denominada de concentração máxima não tóxica (CMNT) e utilizada para os estudos antivirais.

\section{Verificação da viabilidade celular}

Os extratos e frações nas mesmas diluições e condições já descritas foram colocados sobre as monocamadas de células confluentes. $O$ efeito na viabilidade celular foi determinado através da técnica descrita por Neyndorff e colaboradores (1990), com pequenas modificações. A técnica consistiu na incorporação do corante vermelho neutro pelas células vivas e posterior quantificação por leitura em espectrofotômetro, em comprimento de onda de $492 \mathrm{~nm}$. A percentagem de células viáveis foi obtida pela fórmula:

\section{DOdr - DOcontr x 100 \\ DOvn - DOcontr}

sendo calculada a toxidade para $50 \%$ das culturas de células $\left(\mathrm{CC}_{50}\right)$ onde:

-DOdr, Densidade ótica da droga;

-DOcontr, Densidade ótica controle de células

-DOvn, Densidade ótica Vermelho neutro

\section{Avaliação da atividade antiviral}

Suspensão viral em várias diluições foi inoculada em cultura de células na presença dos extratos na CMNT e na ausência dos extratos (controle de vírus), apenas com o meio de cultura sem soro. Ambas as culturas (teste e controle) foram incubadas a $37{ }^{\circ} \mathrm{C}$ por $48 \mathrm{~h}$ em ambiente com $5 \%$ de $\mathrm{CO}_{2}$.

A atividade antiviral foi avaliada pela observação da redução do título viral na presença (teste) e na ausência (controle) dos extratos e frações, determinados por $\mathrm{TCID}_{50} / \mathrm{ml}$ (Reed \& Muench, 1938), sendo calculada a percentagem de inibição (PI), de acordo com a fórmula proposta por Nishimura e colaboradores (1977).

$$
P l=\frac{1-\text { anti } \log T}{\text { anti } \log C} \times 100
$$

onde $\mathrm{T}$ corresponde a unidades infecciosas na cultura de células tratadas com os extratos e frações, e $\mathrm{C}$, unidades infecciosas na cultura de células não tratadas (controle).

\section{RESULTADOS E DISCUSSÃO}

\section{Avaliação da atividade antiviral}

Os extratos etanólico (EEPT), hexânico (EHPT), diclorometânico (EDPT), em acetato de etila (EAPT) e butanólico (EBPT) provenientes das inflorescências coletadas na região de Petrópolis foram testados in vitro para atividade contra o herpesvírus simples humano tipo 1 resistente ao Aciclovir (HSV-1-ACVr) e contra o herpesvírus simples humano tipo 2 resistente ao Aciclovir (HSV-2-ACVr) através da avaliação do índice de inibição viral (IIV) e da respectiva percentagem de inibição (PI).

Da mesma forma foi feita a avaliação com os extratos da planta proveniente da região de Santo Antônio de Pádua (de EEPD a FAPD), sendo que FAPD é a fração aquosa resultante da partição, não testada na amostra de Petrópolis. 
Tabela 1. Citotoxidade e atividade antiviral de inflorescências de Musa acuminata Colla, Musaceae.

\begin{tabular}{|c|c|c|c|c|c|c|}
\hline \multicolumn{7}{|c|}{ Citotoxidade e atividade antiviral } \\
\hline & \multirow{2}{*}{$\mathrm{CMNT}(\mu \mathrm{g} / \mathrm{mL})$} & \multirow{2}{*}{$\mathrm{CC}_{50}(\mu \mathrm{g} / \mathrm{mL})$} & \multicolumn{2}{|c|}{ HSV-1-ACVr } & \multicolumn{2}{|c|}{ HSV-2-ACVr } \\
\hline & & & IIV & PI & IIV & PI \\
\hline EEPT & 100 & $>200$ & 0,88 & 86,8 & 0,38 & 58,3 \\
\hline EHPT & 25 & $>200$ & 1 & 90 & 0,13 & 25,9 \\
\hline EDPT & 25 & $>200$ & 0,63 & 76,5 & 0,23 & 41,1 \\
\hline EAPT & 200 & $>200$ & 0,38 & 58,3 & 1,13 & 92,6 \\
\hline EBPT & 200 & $>200$ & 0,88 & 86,8 & 0,88 & 86,8 \\
\hline EEPD & 25 & $>200$ & 0,63 & 76,5 & 0 & 0 \\
\hline EHPD & 50 & $>200$ & 0,88 & 86,8 & 0,32 & 52,1 \\
\hline EDPD & 50 & $>200$ & 0,63 & 76,5 & 0,27 & 46,3 \\
\hline EAPD & 100 & $>200$ & 1,03 & 90,6 & 0,63 & 76,5 \\
\hline EBPD & 25 & $>200$ & 0,27 & 46,3 & 0,38 & 58,3 \\
\hline FAPD & 50 & $>200$ & 0,38 & 58,3 & 0 & 0 \\
\hline
\end{tabular}

Apesar de todos os extratos terem apresentado a $\mathrm{CC}_{50}$ (concentração citotóxica para $50 \%$ das culturas de células) superior a $200 \mu \mathrm{g} / \mathrm{mL}$, foram empregadas nas experiências as amostras em concentrações nas quais não foram observadas alterações na morfologia celular (CMNT).

As CMNT variaram de 25 a $200 \mu \mathrm{g} / \mathrm{mL}$. Dos onze extratos avaliados, cinco foram capazes de inibir em mais de $80 \%$ a replicação da amostra do herpesvírus simples humano tipos 1 (HSV-1-ACVr). A amostra de herpesvírus simples humano tipo 2 (HSV-2-ACVr) foi inibida por dois extratos em concentrações não citotóxicas.

\section{CONCLUSÃO}

Os estudos com M. acuminata indicam grande potencial inibitório na replicação dos hespesvírus resistente ao Aciclovir. O extrato butanólico das inflorescências de Musa acuminata, coletadas em Petrópolis, foi o único extrato que apresentou inibição para as duas amostras testadas de vírus mostrando inibição superior a $80 \%$, sem apresentar toxidade para as células, na maior concentração empregada $(200 \mu \mathrm{g} / \mathrm{mL})$. Diante desses resultados, estudos posteriores serão realizados para determinar a(s) substância(s) bioativa(s) presente(s) nesses extratos e em qual(is) etapa(s) da síntese viral esses extratos atuam.

\section{REFERÊNCIAS}

Bernardi WF, Rodrigues BI, Neto PC, Ando A, Neto AT, Ceravolo LC, Montes SMNM 2004. Micropropagação de baixo custo em bananeira cv. Maçã em meios com diferentes fontes de carbono e avaliação da performance em campo das mudas produzidas, Jaboticabal-SP. Rev Bras Fruticul 26: 503-506.

Costa MAAM, Brito ARMS 1997. Effects of prolonged administration of Musa paradisiaca L. (banana), an antiulcerogenic substance, in rats. Phytother Res 11: 2831.

Ghosal S, Saini KS 1984. Sitoindosides I and II, two new antiulcerogenic sterylacylglucosides from Musa paradisiaca. J Chem Res-S 4: 110.

Hirai N, Ishida H, Koshimizu K 1994. A phenalenone-type phytoalexin from Musa acuminata. Phytochemistry 37: 383-385.

Kamo T, Hirai N, Iwami K, Fujioka, D, Ohigashi H 2001. New phenylphenalenones from banana fruit. Tetrahedron 57: 7649-7656.

Kanazawa K, Sakakibara H 2000. High content of dopamine, a strong antioxidant, in Cavendish banana. J Agric Food Chem 48: 844-848.

Lewis DA, Fields WN, Shaw GP 1999. A natural flavonoid present in unripe plantain banana pulp (Musa sapientum L. var. paradisiaca) protects the gastric mucosa from aspirininduced erosions. J Ethnopharmacol 65: 283-288.

Luque-Ortega JR, Martinez S, Saugar JM, Izquierdo LR, Abad T, Luis JG, Pinero J, Valladares B, Rivas L 2004. Funguselicited metabolites from plants as an enriched source for new leishmanicidal agents: Antifungal phenylphenalenone phytoalexins from the banana plant (Musa acuminata) target mitochondria of Leishmania donovani promastigotes. Antimicrob Agents Ch 48: 1534-1540.

Neyndorff HC, Bartel DL, Tufaro F, Levy JG 1990. Development of a model to demonstrate photosensitizer-mediated viral inactivation in blood. Transfusion 30: 485-490.

Nishimura T, Toku K, Fukuyasu H 1977. Antiviral compounds. XII. Antiviral activity of aminohydrazones of alkoxyphenyl substituted carbonyl compounds against influenza virus in eggs and mice. Kitasato Arch. Exp. Med. 50: 39-46.

Pascual-Villalobos MJ, Rodriguez B 2006. Constituents of Musa balbisiana seeds and their activity against Cryptolestes pusillus. Biochem Syst Ecol 35: 11-16.

Quinines W, Escobar G, Echeverri F, Torres F, Rosero Y, Arango

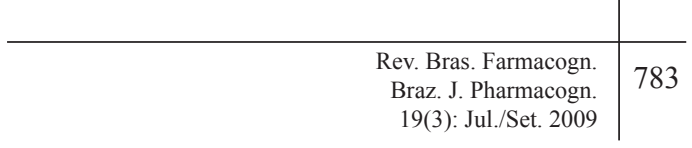


V, Cardona G, Gallego A 2000. Synthesis and antifungal activity of Musa phytoalexins and structural analogs. Molecules 5: 974-980.

Reed LJ, Muench H 1938. A simple method of estimating fifty percents endpoints. Am J Hyg 27: 493-497.

Rodriguez DJ, Chulia J, Simões CMO, Amoros M, Mariotte AM, Girre L 1990. Search for in vitro antiviral activity of a new isoflavonic glycoside from Ulex europaeus. Planta Med 56: 59-62.

Sharma KS, Porwal KM, Metha, BK 1989. In vitro antimicrobial activity of Musa paradisiacal root extracts, Fitoterapia $L X: 157-158$.

Someya SI, Yoshiki Y, Okubo K 2002. Antioxidant compounds from bananas (Musa cavendish). Food Chem 79: 351354.

Talero MMB de, Vejarano AX 1973. Esters with fungistatic activity present in the peel of ripe bananas (Musa paradisiaca). Rev Colombiana Cien Quim-Farm 2: 77103. 